PROCEEDINGS OF THE

AMERICAN MATHEMATICAL SOCIETY

Volume 135, Number 3, March 2007, Pages 895-901

S 0002-9939(06)08508-X

Article electronically published on August 28, 2006

\title{
AVERAGE UNDER THE IWASAWA TRANSFORMATION
}

\author{
MING LIAO AND LONGMIN WANG \\ (Communicated by Ronald A. Fintushel)
}

\begin{abstract}
We derive an averaging property under the Iwasawa decomposition on a semisimple Lie group of noncompact type based on a limiting property of random walks in the Lie group.
\end{abstract}

\section{INTRODUCTION}

Let $G$ be a semisimple Lie group of noncompact type and of finite center with Lie algebra $\mathfrak{g}$, and let $K$ be a maximal compact subgroup with Lie algebra $\mathfrak{k}$. Under this setup, we will use the standard results and notation in Helgason (1978) freely. Thus, $\mathfrak{g}=\mathfrak{k} \oplus \mathfrak{p}$ is the Cartan decomposition of $\mathfrak{g}$ with $\mathfrak{p}$ being an $\operatorname{Ad}(K)$-invariant subspace of $\mathfrak{g}, \mathfrak{a}$ is a maximal abelian subspace of $\mathfrak{p}, \mathfrak{a}_{+}$is a fixed (open) Weyl chamber $(\subset \mathfrak{a})$ and $W$ is the Weyl group which may be regarded as the subgroup of $\operatorname{Ad}(K)$ that leaves $\mathfrak{a}$ invariant. A vector $X$ in $\mathfrak{a}$ is called regular if $w(X) \in \mathfrak{a}_{+}$for some $w \in W$. Let $\mathfrak{n}^{+}$and $\mathfrak{n}^{-}$be respectively the nilpotent Lie subalgebras spanned by positive and negative root spaces, and let $A, N^{+}$and $N^{-}$be respectively the subgroups of $G$ generated by $\mathfrak{a}, \mathfrak{n}^{+}$and $\mathfrak{n}^{-}$. Let $H: G \rightarrow \mathfrak{a}$ and $H^{+}: G \rightarrow \overline{\mathfrak{a}_{+}}$ (the closure of $\mathfrak{a}_{+}$) be respectively the maps defined by the Iwasawa decomposition $G=N^{-} \exp (\mathfrak{a}) K$ and the Cartan decomposition $G=K \exp \left(\overline{\mathfrak{a}_{+}}\right) K$.

For a nonzero $X \in \mathfrak{a}$, Kostant's convexity theorem [3] says that the vector $H\left(k e^{X}\right)$, as $k$ varies over $K$, traces out the convex hull of the $W$-orbit of $X$. In the present paper, we look at the average of this vector under the normalized Haar measure $d k$ on $K$, namely,

$$
\tilde{X}=\int_{K} H\left(k e^{X}\right) d k .
$$

The limiting property of random walks in $G$ to be described later suggests that $\tilde{X}$ may belong to $\mathfrak{a}_{+}$. The purpose of this paper is to prove the following result.

Theorem 1.1. For any nonzero $X \in \mathfrak{a}, \tilde{X} \in \overline{\mathfrak{a}_{+}}$. Moreover, if either the action $\operatorname{Ad}(K)$ on $\mathfrak{p}$ is irreducible or $X$ is regular, then $\tilde{X} \in \mathfrak{a}_{+}$.

We note that the condition in the second part of Theorem 1.1 is necessary because if $\operatorname{Ad}(K)$ is not irreducible on $\mathfrak{p}$, then by Proposition 5.2 in Chapter VIII of [2], $\mathfrak{g}$

Received by the editors April 6, 2005 and, in revised form, September 27, 2005.

2000 Mathematics Subject Classification. Primary 22E46; Secondary 43A80.

Key words and phrases. Iwasawa decomposition.

This paper was completed during the first author's visit to Nankai University and was supported by Nankai University.

(C)2006 American Mathematical Society Reverts to public domain 28 years from publication 
is a direct sum of ideals $\mathfrak{g}_{i}$ such that $\operatorname{Ad}(K)$ leaves invariant each $\mathfrak{p}_{i}=\mathfrak{p} \cap \mathfrak{g}_{i}$ and hence for $X \in \mathfrak{a}_{i}=\mathfrak{a} \cap \mathfrak{p}_{i}, \tilde{X} \in \mathfrak{a}_{i} \cap \overline{\mathfrak{a}_{+}}$, which is part of the boundary of $\mathfrak{a}_{+}$.

In the rank one case $(\operatorname{dim}(\mathfrak{a})=1)$ the result may be derived easily from a simple geometric consideration based on the usual open ball representation of the space $G / K$ with the Cartan and Iwasawa decompositions represented respectively by polar and horospherical coordinates. The general case does not seem to have such an intuition. The rest of this paper is devoted to a description of the random walk connection, the proof of Theorem 1.1 and the computation of $\tilde{X}$ in two special cases, when $X$ is small and when $G=S L(3, \mathbb{R})$.

\section{RANDOM WALKS}

Let $\mu$ be a probability measure on $G$ (defined on Borel subsets of $G$ ). We will write $\mu(f)$ for the integral $\int f(g) \mu(d g)$ whenever it is defined. The convolution of $\mu$ with another measure $\nu$ is defined as usual by $\mu * \nu(f)=\int_{G \times G} f(x y) \mu(d x) \nu(d y)$ for any function $f$ on $G$. Let $z_{j}$ be a sequence of independent $G$-valued random variables with common distribution $\mu$. Define

$$
y_{j}=z_{1} z_{2} \cdots z_{j} \text {. }
$$

The distribution of $y_{j}$ is $\mu^{* j}$, the $j$-fold convolution of $\mu$. The sequence $\left\{y_{j}\right\}$ is called a random walk in $G$ based on $\mu$. Let $y_{j}=\xi_{j} \exp \left(H_{j}^{+}\right) \eta_{j}=n_{j} \exp \left(H_{j}\right) k_{j}$ be respectively the Cartan and the Iwasawa decompositions $G=K \overline{\mathfrak{a}_{+}} K$ and $G=$ $N^{-} A K$.

Let $M$ be the centralizer of $A$ in $K$. By the Bruhat decomposition, $N^{-} M A N^{+}$ is an open subset of $G$, and its complement $\left(N^{-} M A N^{+}\right)^{c}$ is a finite union of lowerdimensional submanifolds of $G$. Any subset $B$ of $G$ is called totally irreducible if there do not exist $g_{1}, g_{2}, \ldots, g_{k}, x \in G$ such that $H \subset \bigcup_{i=1}^{k} g_{i}\left(N^{-} M A N^{+}\right)^{c} x$. A sequence $g_{j}$ in $G$ is called contracting if $\alpha\left(H^{+}\left(g_{j}\right)\right) \rightarrow \infty$ for any positive root $\alpha$.

We will let $G_{\mu}$ and $T_{\mu}$ be respectively the smallest closed group and the smallest closed semigroup containing $\operatorname{supp}(\mu)$. Consider the following conditions:

(i) $G_{\mu}$ is totally irreducible;

(ii) $T_{\mu}$ contains a contracting sequence; and

(iii) $\mu$ has a finite first moment in the sense that $\int_{G}\left\|H^{+}(g)\right\| \mu(d g)<\infty$, where $\|\cdot\|$ is the norm on $\mathfrak{g}$ induced by the Killing form.

In Guivarc'h-Raugi [1, the limiting probability of random walks in $G$ was studied under these conditions, it was proved that almost surely, $n_{j}$ and $\xi_{j} M$ converge as $j \rightarrow \infty$, and

$$
\lim _{j \rightarrow \infty} \frac{1}{j} H_{j}^{+}=\lim _{j \rightarrow \infty} \frac{1}{j} H_{j}=\int_{K} \int_{G} H(k x) \mu(d x) \nu(d k) \in \mathfrak{a}_{+},
$$

where $\nu$ is a probability measure on $K$ satisfying $\nu * \mu=\nu$ and the convolution here is defined via the natural action of $G$ on $K=N^{-} A \backslash G$.

Only the last part of this result will be needed, namely, $\int_{K} \int_{G} H(k x) \mu(d x) \nu(d k) \in$ $\mathfrak{a}_{+}$. This was not proved directly in [1, but rather,

$$
\lim _{j \rightarrow \infty}(1 / j) H_{j}=\int_{K} \int_{G} H(k x) \mu(d x) \nu(d k)
$$

and $\lim _{j \rightarrow \infty}(1 / j) H_{j} \in \mathfrak{a}_{+}$were proved separately by probabilistic and ergodic arguments. 
Note that the result in [1] was stated in a slightly different form but from which the above statement may be derived easily. In [1], the condition (iii) was stated as

$$
\int_{G}|\alpha(H(x))| \mu(d x)<\infty
$$

for a negative root $\alpha$, and the conclusion was

$$
\lim _{j \rightarrow \infty} \frac{1}{j} \alpha\left(H_{j}\right)=\int_{\tilde{B}} \int_{G} \alpha(H(b, x)) \mu(d x) \tilde{\nu}(d b)<0,
$$

where $\tilde{B}=M \backslash K=N^{-} A M \backslash G, H(b, x)=H(k x)$ with $b=M k$ and $k \in K$, and $\tilde{\nu}$ is a probability measure on $\tilde{B}$ satisfying $\tilde{\nu} * \mu=\tilde{\nu}$. However, because $\|H(x)\| \leq\left\|H^{+}(x)\right\|$, (iii) implies (2.3) for any root $\alpha$, and the validity of (2.4) for any negative root $\alpha$ implies the second equality in (2.2).

The distribution $\mu$ is called $K$-conjugate invariant if $c_{k} \mu=\mu$ for any $k \in K$, where $c_{k}$ is the conjugation map: $G \ni g \mapsto k g k^{-1} \in G$ and $c_{k} \mu$ is the measure on $G$ defined by $c_{k} \mu(f)=\mu\left(f \circ c_{k}\right)$ for any function $f$ on $G$. By the Cartan decomposition $G=K \exp \left(\overline{\mathfrak{a}_{+}}\right) K$ and the fact that $H(x k)=H(x)$ for $x \in G$ and $k \in K$, if $\mu$ is $K$-conjugate invariant, then

$$
\int_{K} \int_{G} H(k x) \mu(d x) \nu(d k)=\int_{G} H(x) \mu(d x)=\int_{\overline{\mathfrak{a}}_{+}} \int_{K} H\left(k e^{X}\right) d k \eta(d X),
$$

where $\eta$ is the probability measure $H^{+} \mu$ on $\overline{\mathfrak{a}_{+}}$. Since the expression in (2.5) belongs to $\mathfrak{a}_{+}$, it suggests that $\tilde{X}=\int_{K} H\left(k e^{X}\right) d k$ may be contained in $\mathfrak{a}_{+}$for each $X \in \overline{\mathfrak{a}_{+}}$.

\section{Proof of Theorem 1.1}

Because any $X \in \mathfrak{p}$ is an $\operatorname{Ad}(K)$ image of an element of $\overline{\mathfrak{a}_{+}}$, it suffices to prove Theorem 1.1 for any nonzero $X \in \overline{\mathfrak{a}_{+}}$. Fix such an $X$.

For any probability measure $\eta$ on $\overline{\mathfrak{a}_{+}}$with $\operatorname{supp}(\eta)=\overline{\mathfrak{a}_{+}}$and $\int\|Y\| \eta(d Y)<\infty$, there is a $K$-conjugate invariant probability measure $\mu$ on $G$ satisfying (i), (ii), and (iii) with $\eta=H^{+} \mu$. Since the expression in (2.5) belongs to $\mathfrak{a}_{+}$for any such $\eta$, it follows that $\tilde{X}=\int_{K} H\left(k e^{X}\right) d k \in \overline{\mathfrak{a}_{+}}$. This proves the first part of Theorem 1.1.

Let $\mu$ be defined by

$$
\mu(f)=\int_{K} \int_{K} f\left(k_{1} e^{X} k_{2}\right) d k_{1} d k_{2}
$$

for any function $f$ on $G$. Then $\mu$ is $K$-conjugate invariant with $H^{+} \mu=\delta_{X}$ and hence by (2.5),

$$
\int_{K} \int_{G} H(k x) \mu(d x) \nu(d k)=\int_{K} H\left(k e^{X}\right) d k=\tilde{X} .
$$

It is easy to see that the finite first moment condition (iii) holds. If we can show that (i) and (ii) also hold, then $\tilde{X}$ must belong to $\mathfrak{a}_{+}$. Therefore, to prove Theorem 1.1. it suffices to prove $T_{\mu}=G$ for $\mu$ defined by (3.1).

It is clear that $k e^{w X} \in T_{\mu}$ for $k \in K$ and $w \in W$. Let $w_{0}, w_{1}, \ldots, w_{k}$ be all the elements of the Weyl group $W$ with $w_{0}$ being the identity element. Then $\sum_{i=0}^{k} w_{i} X=0$. It follows that $-X=\sum_{i=1}^{k} w_{i} X$ and hence $e^{-X}=\prod_{i=1}^{k} e^{w_{i} X} \in T_{\mu}$. Moreover, for $k \in K, k=k \exp \left(\sum_{i=0}^{k} w_{i} X\right)=k e^{X} \prod_{i \geq 1} e^{w_{i} X} \in T_{\mu}$. This shows that $K \subset T_{\mu}$. 
For any two smooth $G$-valued functions $\phi(t)$ and $\psi(t)$ defined in an interval $I$ containing 0 , we will write $\phi(t) \approx \psi(t)$ if they differ by $O\left(t^{2}\right)$. Suppose $\phi(t) \in T_{\mu}$ for $t \in I$ and $e^{t Y} \approx \phi(t)$ for some $Y \in \mathfrak{g}$. Since $T_{\mu}$ is a semigroup, $\phi(t)^{k} \in T_{\mu}$ for any integer $k>0$. Note that $e^{t Y}$ for any $t>0$ is a limiting point of a sequence from the set $\left\{\phi(t)^{k}\right\}$ (as $t \rightarrow 0$ and $k \rightarrow \infty$ ). It follows by the closeness of $T_{\mu}$ that $e^{t Y} \in T_{\mu}$ for $t>0$.

Assume $\operatorname{Ad}(K)$ is irreducible on $\mathfrak{p}$. Suppose there is a nonzero $Y \in \mathfrak{p}$ such that $e^{t Y} \in T_{\mu}$ for any $t>0$. The $K$-conjugate invariance of $\mu$ implies that $T_{\mu}$ is invariant under the $K$-conjugation. Thus, $e^{t \operatorname{Ad}(k) Y} \in T_{\mu}$ for any $k \in K$ and $t>0$. The irreducibility of $\operatorname{Ad}(K)$ on $\mathfrak{p}$ implies that the set $\{\operatorname{Ad}(k) Y ; k \in K\}$ contains a basis of $\mathfrak{p}$, say $Y_{1}, Y_{2}, \ldots, Y_{k}$. Any vector of $\mathfrak{p}$ may be written as $\sum_{i=1}^{k} a_{i} Y_{i}$ for some $a_{i} \in \mathbb{R}$. Since $\exp \left(t \sum_{i=1}^{k} a_{i} Y_{i}\right) \approx \prod_{i=1}^{k} \exp \left(t a_{i} Y_{i}\right) \in T_{\mu}$, it follows that $\exp \left(\sum_{i=1}^{k} a_{i} Y_{i}\right) \in T_{\mu}$ and hence $e^{\mathfrak{p}} \subset T_{\mu}$. Because $G=K e^{\mathfrak{p}}$ and $K \subset T_{\mu}, T_{\mu}=G$. Thus, to prove $T_{\mu}=G$ under the irreducibility assumption, it suffices to find a nonzero $Y \in \mathfrak{p}$ such that $e^{t Y} \in T_{\mu}$ for $t>0$.

Since $X \in \overline{\mathfrak{a}_{+}}$is nonzero, there is a positive root $\alpha$ with $\alpha(X)>0$. Let $X_{\alpha}$ be a nonzero root vector of $\alpha$ and let $X_{\alpha}=Y_{\alpha}+Z_{\alpha}$ be the decomposition $\mathfrak{g}=\mathfrak{p} \oplus \mathfrak{k}$. Then $\left[X, X_{\alpha}\right]=\alpha(X) X_{\alpha},\left[X, Y_{\alpha}\right]=\alpha(X) Z_{\alpha}$ and $\left[X, Z_{\alpha}\right]=\alpha(X) Y_{\alpha}$. Note that $\exp \left[\operatorname{Ad}\left(e^{t Z_{\alpha}}\right) X\right] \in T_{\mu}$ for $t \in \mathbb{R}$. Since $\left.(d / d t) \operatorname{Ad}\left(e^{t Z_{\alpha}}\right) X\right|_{t=0}=\left[Z_{\alpha}, X\right]=-\alpha(X) Y_{\alpha}$,

$$
\exp \left[\operatorname{Ad}\left(e^{t Z_{\alpha}}\right) X\right]=\exp \left[X-t \alpha(X) Y_{\alpha}+O\left(t^{2}\right)\right] \approx \exp \left[X-t \alpha(X) Y_{\alpha}\right]
$$

By Theorem 1.7 in Chapter 1 of [2], with $\operatorname{ad}(X) Y=[X, Y]$,

$$
\begin{aligned}
& e^{X-t \alpha(X) Y_{\alpha}} \approx e^{X} \exp \left\{\left[\frac{1-e^{-\operatorname{ad}(X)}}{\operatorname{ad}(X)}\right]\left[-t \alpha(X) Y_{\alpha}\right]\right\} \\
= & e^{X} \exp \left\{-t \alpha(X) \sum_{i=0}^{\infty} \frac{(-1)^{i}}{(i+1) !} \operatorname{ad}(X)^{i} Y_{\alpha}\right\} \\
= & e^{X} \exp \left\{t[\cosh \alpha(X)-1] Z_{\alpha}-t[\sinh \alpha(X)] Y_{\alpha}\right\} \\
\approx & e^{X} e^{t[\cosh \alpha(X)-1] Z_{\alpha}} e^{-t[\sinh \alpha(X)] Y_{\alpha}} .
\end{aligned}
$$

Let $k=e^{t[\cosh \alpha(X)-1] Z_{\alpha}} \in K$ and $a=\sinh \alpha(X)>0$. Since

$$
\begin{aligned}
e^{-t a Y_{\alpha}} & =k^{-1} e^{-X} e^{X} k e^{-t a Y_{\alpha}} \approx k^{-1} e^{-X} e^{X-t \alpha(x) Y_{\alpha}} \\
& \approx k^{-1} e^{-X} \exp \left[\operatorname{Ad}\left(e^{t Z_{\alpha}}\right) X\right] \in T_{\mu},
\end{aligned}
$$

it follows that $e^{-t Y_{\alpha}} \in T_{\mu}$ for $t>0$. Since $-Y_{\alpha}$ is a nonzero vector in $\mathfrak{p}$, by the discussion in the previous paragraph, this proves $T_{\mu}=G$ under the irreducibility assumption.

Now assume $X \in \mathfrak{a}_{+}$but not the irreducibility of $\operatorname{Ad}(K)$ on $\mathfrak{p}$. The computation in the last paragraph shows that $\exp \left(t Y_{\alpha}\right) \in T_{\mu}$ for any positive root $\alpha$ and $t>0$. Since $\exp \left(t X_{\alpha}\right)=\exp \left[t\left(Y_{\alpha}+Z_{\alpha}\right)\right] \approx \exp \left(t Y_{\alpha}\right) \exp \left(t Z_{\alpha}\right) \in T_{\mu}$, it follows that $N^{+} \in$ $T_{\mu}$. Using $G=K N^{+} K$, we may conclude $T_{\mu}=G$. Theorem 1.1 is completely proved.

\section{Computation of $\tilde{X}$}

Let $X \in \mathfrak{a}$ with $X \neq 0$ as before. In this section, we will compute $\tilde{X}$ defined by (1.1) in two special cases: when $X$ is small and when $G=S L(3, \mathbb{R})$, the group of 
$3 \times 3$ real matrices of unit determinant, which is perhaps the simplest semisimple Lie group of rank $>1$.

Consider the function $\phi(t)=\int_{K} H\left(k e^{t X}\right) d k$ for $t \in \mathbb{R}$. Then $\phi(0)=0$. For any $g \in G$ and $Y \in \mathfrak{g}$, we will write $g=g_{N} g_{A} g_{K}$ and $Y=Y_{\mathfrak{n}}+Y_{\mathfrak{a}}+Y_{\mathfrak{k}}$ respectively for the decompositions $G=N^{-} A K$ and $\mathfrak{g}=\mathfrak{n}^{-} \oplus \mathfrak{a} \oplus \mathfrak{k}$. Note that $g_{A}=H(g)$, $H\left(g e^{Y}\right)=H(g)+H\left(g_{K} e^{Y}\right)$ and for $k \in K, H\left(k e^{t Y}\right)=H\left(e^{t \operatorname{Ad}(k) Y}\right) \approx e^{t[\operatorname{Ad}(k) Y]_{\mathfrak{a}}}$. Thus,

$$
\begin{array}{r}
\phi^{\prime}(t)=\left.\frac{d}{d s} \int_{K} H\left(k e^{(t+s) X}\right) d k\right|_{s=0}=\left.\frac{d}{d s} \int_{K} H\left(k e^{t X} e^{s X}\right) d k\right|_{s=0} \\
=\int_{K}\left[\operatorname{Ad}\left(\left(k e^{t X}\right)_{K}\right) X\right]_{\mathfrak{a}} d k, \\
\left.\phi^{\prime}(0)=\int_{K}[\operatorname{Ad}(k) X]_{\mathfrak{a}} d k=0 \quad \text { (because } \forall Y \in \mathfrak{p}, \int_{K} \operatorname{Ad}(k) Y d k=0\right),
\end{array}
$$

and

$$
\begin{aligned}
\phi^{\prime \prime}(0) & =\left.\frac{d}{d t} \int_{K}\left[\operatorname{Ad}\left(\left(e^{t \operatorname{Ad}(k) X}\right)_{K} k\right) X\right]_{\mathfrak{a}} d k\right|_{t=0} \\
& =\left.\frac{d}{d t} \int_{K}\left[\operatorname{Ad}\left(e^{t[\operatorname{Ad}(k) X]_{\mathfrak{k}}}\right) \operatorname{Ad}(k) X\right]_{\mathfrak{a}} d k\right|_{t=0} \\
& =\int_{K}\left[[\operatorname{Ad}(K) X]_{\mathfrak{k}}, \operatorname{Ad}(k) X\right]_{\mathfrak{a}} d k .
\end{aligned}
$$

Let $\alpha_{1}, \ldots, \alpha_{r}$ be the complete list of positive roots, each repeated as many times as its multiplicity, let $\rho=(1 / 2) \sum_{i=1}^{r} \alpha_{i}$ be the half sum of positive roots, and let $H_{\rho}$ be the element of $\mathfrak{a}$ representing $\rho$ in the sense that $\rho(Y)=\left\langle Y, H_{\rho}\right\rangle$ for $Y \in \mathfrak{a}$, where $\langle\cdot, \cdot\rangle$ is the inner product on $\mathfrak{g}$ induced by the Killing form. By Lemmas 7.3 and 7.4 in [4, under the assumption that the action $\operatorname{Ad}(K)$ on $\mathfrak{p}$ is irreducible,

$$
\phi^{\prime \prime}(0)=\int_{K}\left[[\operatorname{Ad}(k) X]_{\mathfrak{k}}, \operatorname{Ad}(k) X\right]_{\mathfrak{a}} d k=\frac{2}{p}\|X\|^{2} H_{\rho},
$$

where $p=\operatorname{dim}(\mathfrak{p})$. Since $\phi(t)=(t X)^{\sim}$, writing $X$ for $t X$, we obtain the following estimate for $\tilde{X}$ when $\|X\|$ is small:

$$
\tilde{X}=\frac{1}{p}\|X\|^{2} H_{\rho}+O\left(\|X\|^{3}\right) .
$$

Now let $G=S L(3, \mathbb{R})$ and $X=\operatorname{diag}(\lambda, \lambda,-2 \lambda)$ for some $\lambda>0$. In this case, $K$ is the group $S O(3)$ of $3 \times 3$ orthogonal matrices of unit determinant, $\mathfrak{a}$ is the space of traceless $3 \times 3$ real diagonal matrices, $\mathfrak{a}_{+}$is the set consisting of those matrices in $\mathfrak{a}$ with strictly descending diagonal, and $X$ given above belongs to the boundary of $\mathfrak{a}_{+}$.

The centralizer of $X$ in $K=S O(3)$ is the subgroup $\operatorname{diag}\{S O(2), 1\}$ of rotations that fix the vector $z=(0,0,1)^{\prime} \in \mathbb{R}^{3}$. The orbit of $z$ under the action of $K$ is the unit sphere in $\mathbb{R}^{3}$ which may be parametrized as $R_{z}(\phi) R_{y}(\theta) z$ for $0 \leq \theta \leq \pi$ and $0 \leq \phi \leq 2 \pi$, where $R_{y}(\phi)$ and $R_{z}(\phi)$ denote respectively the matrices in $S O(3)$ that rotate around the $y$ and $z$ axes by an angle $\phi$. Because the normalized Haar measure $d k$ on $K=S O(3)$ induces the uniform distribution on the unit sphere given by $\sin \theta d \theta d \phi /(4 \pi)$, it follows that

$$
\tilde{X}=\frac{1}{4 \pi} \int_{0}^{2 \pi} \int_{0}^{\pi} H\left(R_{z}(\phi) R_{y}(\theta) e^{X}\right) \sin \theta d \theta d \phi .
$$


We have, by a simple computation,

$$
\begin{aligned}
R_{z}(\phi) R_{y}(\theta) e^{X} & =\left[\begin{array}{lll}
\cos \phi & -\sin \phi & 0 \\
\sin \phi & \cos \phi & 0 \\
0 & 0 & 1
\end{array}\right]\left[\begin{array}{lll}
\cos \theta & 0 & \sin \theta \\
0 & 1 & 0 \\
-\sin \theta & 0 & \cos \theta
\end{array}\right]\left[\begin{array}{lll}
e^{\lambda} & 0 & 0 \\
0 & e^{\lambda} & 0 \\
0 & 0 & e^{-2 \lambda}
\end{array}\right] \\
& =\left[\begin{array}{lll}
e^{\lambda} \cos \theta \cos \phi & -e^{\lambda} \sin \phi & e^{-2 \lambda} \sin \theta \cos \phi \\
e^{\lambda} \cos \theta \sin \phi & e^{\lambda} \cos \phi & e^{-2 \lambda} \sin \theta \sin \phi \\
-e^{\lambda} \sin \theta & 0 & e^{-2 \lambda} \cos \theta
\end{array}\right] .
\end{aligned}
$$

Note that $H\left(R_{z}(\phi) R_{y}(\theta) e^{X}\right)$ is a diagonal matrix

$$
\operatorname{diag}\left(h_{1}(\theta, \phi), h_{2}(\theta, \phi), h_{3}(\theta, \phi)\right)
$$

with $\sum_{i=1}^{3} h_{i}(\theta, \phi)=0$. To determine this matrix, let $r_{1}, r_{2}, r_{3}$ be the three rows of the matrix $\left[R_{z}(\phi) R_{y}(\theta) e^{X}\right]$ and find a lower triangular matrix $\left\{s_{i j}\right\}$ of unit determinant such that multiplying $\left\{s_{i j}\right\}$ to the left of $R_{z}(\phi) R_{y}(\theta) e^{X}$ will yield an orthogonal matrix. Using the Gram-Schmidt orthogonalization, we obtain

$$
s_{11}=\left|r_{1}\right|^{-1}, \quad s_{22}=\frac{\left|r_{1}\right|}{\sqrt{\left|r_{1}\right|^{2}\left|r_{2}\right|^{2}-\left(r_{1} \cdot r_{2}\right)^{2}}}, \quad s_{21}=-s_{22} \frac{\left(r_{1} \cdot r_{2}\right)}{\left|r_{1}\right|^{2}},
$$

where $|r|$ and $\left(r_{1} \cdot r_{2}\right)$ denote the usual norm and inner product on $\mathbb{R}^{3}$. Because of the requirement of a unit determinant, $s_{33}=\sqrt{\left|r_{1}\right|^{2}\left|r_{2}\right|^{2}-\left(r_{1} \cdot r_{2}\right)^{2}}$. Since $\exp \left(h_{i}\right)$ is just the reciprocal of $s_{i i}$,

$$
\begin{gathered}
\exp \left(h_{1}\right)=\left|r_{1}\right|=\sqrt{e^{2 \lambda}-\left(e^{2 \lambda}-e^{-4 \lambda}\right) \sin ^{2} \theta \cos ^{2} \phi}, \\
\exp \left(h_{3}\right)=\frac{1}{\sqrt{\left|r_{1}\right|^{2}\left|r_{2}\right|^{2}-\left(r_{1} \cdot r_{2}\right)^{2}}}=\frac{1}{e^{\lambda} \sqrt{e^{-4 \lambda}+\left(e^{2 \lambda}-e^{-4 \lambda}\right) \cos ^{2} \theta}}
\end{gathered}
$$

and $\exp \left(h_{2}\right)=1 /\left[\exp \left(h_{1}\right) \exp \left(h_{3}\right)\right]$.

We have

$$
\tilde{X}=\operatorname{diag}\left(\tilde{h}_{1}, \tilde{h}_{2}, \tilde{h}_{3}\right) \quad \text { with } \quad \tilde{h}_{i}=\frac{1}{4 \pi} \int_{0}^{2 \pi} \int_{0}^{\pi} h_{i}(\theta, \phi) \sin \theta d \theta d \phi .
$$

Using the integral formula $(a, b>0)$ :

$$
\begin{aligned}
& \int \ln \left(a \pm b x^{2}\right) d x \\
& \quad=x \ln \left(a \pm b x^{2}\right)-2 x+\left\{\begin{array}{l}
2 \sqrt{a / b} \tan ^{-1}(\sqrt{b / a} x)+C( \pm=+) \\
\sqrt{a / b} \ln [(\sqrt{a}+\sqrt{b} x) /(\sqrt{a}-\sqrt{b} x)+C \quad( \pm=-)
\end{array}\right.
\end{aligned}
$$

we obtain

$$
\begin{aligned}
& \tilde{h}_{1}=\frac{1}{8 \pi} \int_{0}^{2 \pi} \int_{0}^{\pi} \ln \left[e^{2 \lambda}-\left(e^{2 \lambda}-e^{-4 \lambda}\right) \sin ^{2} \theta \cos ^{2} \phi\right] \sin \theta d \theta d \phi \\
& =\frac{1}{8 \pi} \int_{0}^{2 \pi} \int_{0}^{\pi} \ln \left[e^{2 \lambda}-\left(e^{2 \lambda}-e^{-4 \lambda}\right) \cos ^{2} \theta\right] \sin \theta d \theta d \phi \quad \text { (by spherical symmetry, }
\end{aligned}
$$

noting $x=\sin \theta \cos \phi$ and $z=\cos \theta$ in spherical coordinates)

$$
\begin{aligned}
& \left.=\frac{1}{2} \int_{0}^{1} \ln \left[e^{2 \lambda}-\left(e^{2 \lambda}-e^{-4 \lambda}\right) x^{2}\right] d x \quad \text { (by substitution } x=\cos \theta\right) \\
& =-2 \lambda-1+\frac{1}{2 \sqrt{1-e^{-6 \lambda}}} \ln \frac{1+\sqrt{1-e^{-6 \lambda}}}{1-\sqrt{1-e^{-6 \lambda}}}
\end{aligned}
$$


and

$$
\begin{aligned}
& \tilde{h}_{3}=-\frac{1}{4 \pi} \int_{0}^{2 \pi} \int_{0}^{\pi}\left\{\lambda+\frac{1}{2} \ln \left[e^{-4 \lambda}+\left(e^{2 \lambda}-e^{-4 \lambda}\right) \cos ^{2} \theta\right]\right\} \sin \theta d \theta d \phi \\
= & \left.-\lambda-\frac{1}{2} \int_{0}^{1} \ln \left[e^{-4 \lambda}+\left(e^{2 \lambda}-e^{-4 \lambda}\right) x^{2}\right] d x \quad \text { (by substitution } x=\cos \theta\right) \\
= & -2 \lambda+1-\frac{1}{\sqrt{e^{6 \lambda}-1}} \tan ^{-1}\left(\sqrt{e^{6 \lambda}-1}\right) .
\end{aligned}
$$

By (4.2), (4.3), (4.4) and $\tilde{h}_{2}=-\tilde{h}_{1}-\tilde{h}_{3}$, the vector $\tilde{X}$ is now explicitly determined. By Theorem 1.1, $\tilde{X} \in \mathfrak{a}_{+}$. In the present case, this means $\tilde{h}_{1}>\tilde{h}_{2}>\tilde{h}_{3}$, which may be verified directly by a numerical computation. The plot generated by the computation also demonstrates the estimate (4.1) and shows that $\tilde{X}$ is not a multiple of $H_{\rho}=\operatorname{diag}(1 / 6,0,-1 / 6)$.

\section{REFERENCES}

1. Y. Guivarc'h et A. Raugi, Frontière de Furstenberg, propriétés de contraction et théorèmes de convergence, Z. Wahrsch. verw. Gebiete 69 (1985), 187-242. MR0779457 (86h:60126)

2. S. Helgason, Differential geometry, Lie groups, and symmetric spaces, Academic Press, 1978. MR0514561 (80k:53081)

3. B. Kostant, On convexity, the Weyl group and the Iwasawa decomposition, Ann. Sci. École Norm. Sup. 6 (1973), 413-455. MR0364552 (51:806)

4. M. Liao, Lévy processes in Lie groups, Cambridge Univ. Press, 2004. MR2060091 (2005e:60004)

Department of Mathematics, Auburn University, Auburn, Alabama 36849

E-mail address: liaomin@auburn.edu

Department of Mathematics, Nankai University, Tianjin, People's Republic of China

E-mail address: wanglm@nankai.edu.cn 